\title{
Construcción y propiedades psicométricas de la Escala de Ansiedad de Lima de 20 ítems (EAL-20).
}

\author{
Construction and psychometric properties of 20-item Lima Anxiety Scale (LAS-20). \\ Antonio Lozano-Vargas ${ }^{1,2, a, c,}$, Johann M. Vega-Dienstmaier ${ }^{1, a, b}$
}

\section{RESUMEN}

Objetivo: Seleccionar los ítems más representativos de una escala de ansiedad de 130 ítems (Escala de Ansiedad de Lima, EAL-130) para hacer posible la construcción de una versión breve de 20 ítems (EAL-20). Métodos: A partir de los datos del estudio previo de validación de la EAL-130 en 254 pacientes, se utilizó el método de análisis de regresión múltiple para identificar los ítems que mejor predecían los puntajes totales de cada una de 5 subescalas correspondientes a 5 síndromes incluidos en la escala original (ansiedad psíquica, síntomas físicos, pánico, agorafobia y fobia social). Se condujo un análisis factorial de matriz policórica de la nueva escala así generada (EAL-20). Resultados: La EAL-20 tuvo un alfa de Cronbach de 0,8885 y sus 20 ítems cubrieron el 90,38\% de la varianza del puntaje total de la EAL-130. En la EAL-20 se identificaron 4 factores correspondientes a ansiedad física, fobia social, ansiedad psíquica y agorafobia. Con un punto de corte de $\geq 10$ se obtuvo un equilibrio entre sensibilidad y especificidad para la identificación de un CGI-S $\geq 4$. Conclusiones: Los 20 ítems seleccionados para la EAL-20 permiten evaluar 4 dimensiones de ansiedad, con una mínima pérdida de información respecto a la EAL-130; se sugiere usar un punto de corte $\geq 10$ como indicador de ansiedad moderada o de severidad mayor.

PALABRAS CLAVE: Psicometría, ansiedad, trastornos fóbicos.

\section{SUMMARY}

Objective: To select the most representative items of a 130-item anxiety scale (Lima Anxiety Scale, LAS-130) in order to construct a shorter version of 20 items (LAS-20). Methods: Based on the data of the 254 patients included in the previous validation study of LAS-130, multiple regression analysis was used to identify the items that best predicted the total scores of each of 5 subscales corresponding to 5 syndromes included in the original scale (psychic anxiety, physical symptoms, panic, agoraphobia and social phobia). In addition, a factorial analysis of the polychoric matrix of the new scale (LAS-20) was performed. Results: LAS-20 had a Cronbach's alpha of 0.8885 , and its 20 items covered $90.38 \%$ of the total score variance of LAS-130. In LAS-20, 4 factors were identified corresponding to physical anxiety, social phobia, psychic anxiety and agoraphobia. Using a cut-off score of $\geq 10$, equilibrium between sensitivity and specificity was obtained for detection of a CGI-S $\geq 4$. Conclusions: The 20 items selected for the LAS-20 allow to evaluate 4 anxiety dimensions with a minimum loss of information when compared to the LAS130. A cut-off score of $\geq 10$ is suggested as an indicator of anxiety of moderate or higher severity.

KEYWORDS: Psychometrics, anxiety, phobic disorders.

\footnotetext{
Universidad Peruana Cayetano Heredia, Facultad de Medicina Alberto Hurtado, Sección de Psiquiatría y Salud Mental. Lima, Perú.

2 Hospital Cayetano Heredia, Servicio de Neuro-Psiquiatría. Lima, Perú.

a Médico Psiquiatra.

b Maestría en epidemiología clínica.

c Clínica Anglo Americana. Lima, Perú.
} 


\section{INTRODUCCIÓN}

Los trastornos de ansiedad son uno de los problemas más comunes de salud mental en la población general y con mucha frecuencia son evaluados y tratados en la atención primaria de salud. La prevalencia de vida de los trastornos de ansiedad llega alrededor de un $29 \%$ en los Estados Unidos; y los pacientes que los padecen experimentan una menor calidad de vida, disminución de su productividad y una mayor necesidad del uso de servicios de salud (1).

En el Perú, en un estudio realizado en Lima Metropolitana y la Provincia Constitucional del Callao en el año 2012 se observó que los trastornos de ansiedad presentan una prevalencia de vida de $10,5 \%$, una prevalencia anual de $2,9 \%$, una prevalencia de 6 meses de 2,4\% y una prevalencia actual de 1,9\% (2).

Por otro lado, los trastornos de ansiedad son muy frecuentes en los pacientes que acuden a la atención psiquiátrica ambulatoria; así en un hospital general de Lima Metropolitana se encontraron los siguientes trastornos: trastorno de ansiedad generalizada en un $23,6 \%$, trastorno de pánico en un $13,8 \%$, agorafobia en un $4,7 \%$ y fobia social en un $6,3 \%$ (3).

Desafortunadamente, a pesar de que los trastornos de ansiedad son problemas muy frecuentes de salud mental en nuestra población, no son diagnosticados oportunamente y el tratamiento es muchas veces postergado. Es por ello que existe un creciente interés en los grupos de investigación de salud mental en la elaboración y validación de instrumentos psicométricos con el fin de contar con herramientas que faciliten la identificación de trastornos mentales en la población (4).

Así, en un trabajo inicial se construyó una nueva escala de ansiedad en español de 130 ítems (Escala de Ansiedad de Lima de 130 ítems: EAL-130) a partir de la revisión del contenido de 25 instrumentos para la evaluación de la sintomatología ansiosa en general y de trastornos específicos, incluyendo ansiedad generalizada, pánico, agorafobia, fobia social, trastorno obsesivo-compulsivo y trastorno de estrés postraumático (4).

Por otro lado, en un siguiente estudio realizado en pacientes psiquiátricos ambulatorios se evaluaron las propiedades psicométricas de la EAL-130 y se generó una versión más corta de 72 ítems (EAL-72) (3). Sin embargo, la necesidad de contar con un instrumento más breve que permita identificar la presencia de síntomas de ansiedad en nuestra población motivó la realización del presente trabajo que tiene la finalidad de seleccionar 20 ítems de los 130 originales que puedan predecir adecuadamente los puntajes totales de la EAL-130.

\section{MATERIAL Y MÉTODOS}

En el presente estudio se utilizaron los datos recolectados de 254 pacientes del estudio previo de validación de la EAL-130 realizado por Lozano-Vargas y Vega-Dienstmaier (3). Los pacientes procedían de la consulta ambulatoria de psiquiatría del Hospital Cayetano Heredia (distrito de San Martín de Porres, Lima - Perú); eran de ambos sexos, de 18 a 60 años de edad y con al menos primaria completa. Se excluyeron a los individuos con síntomas psicóticos, demencia o retardo mental.

El proyecto de investigación original (3) recibió la aprobación de los comités de ética de la Universidad Peruana Cayetano Heredia y el Hospital Cayetano Heredia. Todos los individuos aceptaron participar en el estudio y firmaron el consentimiento informado correspondiente.

Para el análisis de datos se utilizó el programa estadístico STATA versión 14. El proceso se llevó a cabo de la siguiente manera:

Cálculo del puntaje de cada subescala: en la escala original de 130 ítems se pueden identificar 7 tipos de síntomas correspondientes a: ansiedad psíquica, síntomas físicos, pánico, agorafobia, fobia social, síndrome obsesivo-compulsivo y trastorno de estrés postraumático. Sólo se calculó la sumatoria de los ítems de cada una de las primeras 5 subescalas; no se incluyeron los ítems sobre síntomas obsesivocompulsivos ni de estrés postraumático ya que estos trastornos ya no forman parte de los trastornos de ansiedad en el DSM-5 (5).

Identificación de los items más relevantes de cada subescala: para cada subescala, mediante regresión lineal múltiple se evaluó la capacidad de cada uno de sus ítems (variables independientes) para predecir su puntaje total (variable dependiente). Luego, se seleccionaron los ítems más representativos de la subescala los cuales en su conjunto eran capaces de explicar al menos el $80 \%$ de varianza de su puntaje total. 
Selección de los 20 items de la escala final: Asimismo, utilizando un procedimiento similar, partiendo de los ítems de todas las subescalas seleccionados en el paso anterior (variables independientes) se escogieron los 20 ítems que mejor predecían el puntaje total de la EAL-130 (variable dependiente). De esta manera, estos 20 ítems constituyeron la EAL-20.

Evaluación de la consistencia interna: se calculó el alfa de Cronbach para la EAL-20. Para cada uno de sus 20 ítems se determinó la correlación con el puntaje total y el alfa de Cronbach si fuera retirado.

Análisis factorial de matriz policórica de la EAL20: se realizó empleando el método de rotación de varimax.

Descripción de los puntajes de la EAL-20: se realizó en función del grado de intensidad de la ansiedad de acuerdo a la Escala de Impresión Clínica Global de Severidad (Clinical Global Impression: CGI-S) (6). La CGI-S es un instrumento hetero administrado de fácil aplicación a través de una entrevista clínica, que permite clasificar la severidad del trastorno del paciente en 7 grados: 1 (normal, sin ansiedad), 2 (mínimamente ansioso), 3 (levemente ansioso), 4 (moderadamente ansioso), 5 (marcadamente ansioso), 6 (gravemente ansioso) y 7 (extremadamente ansioso).

Análisis de la curva ROC de los puntajes de la $E A L-20$ : se realizó tomando como patrón de referencia un nivel severidad de ansiedad de moderado o más de acuerdo a la CGI-S (CGI-S $\geq 4$ ). De este modo se calculó la sensibilidad (s), especificidad (e) y el índice de Youden ( $\mathrm{J}, \mathrm{J}=\mathrm{s}+\mathrm{e}-1)$ para cada punto de corte de la EAL-20.

\section{RESULTADOS}

En la tabla 1 se muestran los coeficientes de regresión de los ítems más asociados a cada subescala y que logran explicar al menos el $80 \%$ de su varianza. Aquí se puede observar superposición entre los síntomas físicos y de pánico.

El alfa de Cronbach de la EAL-20 fue de 0,8885. En la tabla 2 se muestran las características de los 20 ítems seleccionados: el coeficiente de regresión para la predicción del puntaje total de la escala de 130 ítems, la correlación ítem-total y el alfa de Cronbach si el ítem es eliminado. Se puede observar que el alfa de Cronbach disminuye ante el retiro de cada uno de los 20 ítems.
Los resultados de la regresión indican que los 20 ítems seleccionados explican el 90,38\% de la varianza del puntaje total de la escala de 130 ítems; y que los 2 ítems que más aportan a la predicción de dicho puntaje son "Tengo miedo de hacer el ridículo y sentirme humillado o avergonzado" y "Tengo miedo de conocer gente nueva", ambos pertenecientes a la dimensión de fobia social.

En la tabla 3 se muestran los resultados del análisis factorial policórico de la EAL-20, considerando modelos con 1, 2, 3 y 4 factores. Cuando se toman 2 factores se puede notar una dimensión de ansiedad física y psíquica (factor 1) y otra de fobias (factor 2); al considerar 3 factores, las dimensiones resultantes se relacionan con ansiedad física y psíquica (factor 1), fobia social (factor 2) y agorafobia (factor 3); finalmente, con 4 factores podemos observar las dimensiones de ansiedad física (factor 1), fobia social (factor 2), ansiedad psíquica (factor 3) y agorafobia (factor 4). Aunque el "Eigen value" correspondiente al factor 4 es bajo $(0,94)$, es importante considerar el modelo de 4 factores porque permite diferenciar dimensiones que teóricamente son importantes: la ansiedad psíquica de la física.

En la tabla 4 se describen los puntajes de la EAL-20 en función de los niveles de severidad de la ansiedad de acuerdo a la CGI-S.

En la tabla 5 se muestra el análisis de la curva ROC de los puntajes de la EAL-20 tomando como patrón de referencia una CGI-S de 4 ó más (severidad moderada o superior), encontrándose que el equilibrio entre sensibilidad y especificidad se obtiene con un punto de corte de $\geq 10$ y el mayor índice de Youden con un punto de corte de $\geq 11$.

\section{DISCUSIÓN}

El análisis factorial de la escala permite identificar 4 dimensiones: fobia social, ansiedad psíquica, síntomas físicos y agorafobia. Los cuales pueden asemejarse a los 4 factores observados en el estudio de psicometría realizado con el Screen for Adult Anxiety Related Disorders (SCAARED): ansiedad social, ansiedad generalizada, síntomas somáticos/ pánico/agorafobia y ansiedad de separación (7). Asimismo, hay correspondencia entre las dimensiones de la EAL-20 y determinados síndromes encontrados en un estudio de la estructura de la sintomatología mental en pacientes psiquiátricos ambulatorios, estos son: fobia social, ansiedad general, pánico/síntomas 
Construcción y propiedades psicométricas de la Escala de Ansiedad de Lima de 20 ítems (EAL-20).

Tabla 1. Coeficientes de regresión de los ítems capaces de explicar al menos el $80 \%$ de la varianza de cada subescala (ítems más representativos de cada subescala).

Subescala

Coeficiente de regresión (IC 95\%)

para la subescala correspondiente

Fobia social $\left(r^{2}\right.$ aj $\left.=0,8120\right)$

84 -Tengo miedo de conocer gente nueva

$4,25(2,79-5,7)$

28 - Me incomoda acercarme a un grupo de personas e interactuar con ellas

$3,46(2,24-4,68)$

118 - Exámenes, entrevista de trabajo

$3,33(2,18-4,48)$

96 - He tenido miedo de hacer cosas delante de personas que me pueden estar observando

$2,62(1,42-3,82)$

34 - Me es difícil hablar con otras personas, tengo temor, me cuesta iniciar una conversación o no se me ocurre qué decir

$3,14(1,90-4,38)$

80 - Ser observado al trabajar, escribir, caminar, etc.

$2,80(1,64-3,96)$

88 - Tengo miedo de hacer el ridículo y sentirme humillado o avergonzado

$2,78(1,68-3,87)$

Ansiedad psíquica (r2 aj=0,8001)

121 - Miedo sin motivo

$2,68(1,51-3,86)$

081 - Aturdido o confundido

$3,78(2,62-4,94)$

014 - Nervioso

$2,88(1,62-4,13)$

019 - Angustiado-nervios de punta

$2,41(1,22-3,60)$

043 -Agitado-alterado

$3,25(2,12-4,38)$

013 - Cómodo

$3,46(2,32-4,59)$

001- Con facilidad me siento asustado, con temor o miedo

$2,95(1,79-4,10)$

Agorafobia $\left(r^{2}\right.$ aj $\left.=0,8616\right)$

119 - Alejarse de casa

$1,81(1,38-2,25)$

075 - Lugar lleno de gente

$2,15(1,75-2,55)$

117 - Me da miedo caminar por la calle, especialmente por las avenidas grandes

$2,11(1,66-2,55)$

095 - Me da miedo estar en cines, teatros o estadios

$2,53(1,99-3,06)$

127 - Lugares altos

$1,87(1,50-2,23)$

102 - Me dan miedo los supermercados o tiendas

$1,61(0,92-2,29)$

Síntomas físicos $\left(r^{2} a j=0,8093\right)$

021 - Disnea

$3,82(2,78-4,85)$

051 - Dolor o presión en pecho

$3,32(2,27-4,37)$

079-Escalofríos

$3,07(1,99-4,16)$

032 - He sentido tensión, molestias o dolores en los músculos, especialmente en el cuello, hombros y espalda.

$2,78(1,70-3,87)$

104 - Siento debilidad, especialmente en las piernas

$3,28(2,31-4,25)$

045 - Parestesias

$3,80(2,88-4,73)$

Pánico $\left(r^{2}\right.$ aj=0,8055)

021 - Disnea

$3,06(2,33-3,80)$

051 - Dolor o presión en pecho

$3,00(2,29-3,71)$

074 - Caer

$3,72(3,08-4,37)$

045 - Parestesias

$3,00(2,36-3,64)$

$\mathrm{r}^{2} \mathrm{aj}=\mathrm{r}$ cuadrado ajustado. 
Tabla 2. Características de los 20 ítems seleccionados (escala final EAL-20): consistencia interna y su capacidad para predecir el puntaje total de la EAL-130.

\begin{tabular}{|c|c|c|c|}
\hline Ítem & $\begin{array}{l}\text { Coeficiente de regresión (IC } \\
\text { 95\%)(predicción de la EAL- } \\
130 \text { ) }\end{array}$ & $\begin{array}{l}\text { Correlación ítem- } \\
\text { total (EAL-20) }\end{array}$ & $\begin{array}{l}\text { Alfa si el ítem es } \\
\text { eliminado (EAL-20) }\end{array}$ \\
\hline
\end{tabular}

84-Tengo miedo de conocer gente nueva

$$
6,25(2,75-9,75)
$$

$4,06(1,22-6,89)$

una entrevista de trabajo

034-Me es difícil hablar con otras personas, tengo temor, me cuesta iniciar una conversación o no se me ocurre qué decir

080-Me incomoda que me observen cuando trabajo, escribo, camino o tengo algún objeto que se me puede caer

088-Tengo miedo de hacer el ridículo y sentirme humillado o avergonzado

119-Me da miedo alejarme de mi casa

75-Me incomodaría entrar o salir de un lugar lleno de gente.

127-Me da miedo estar en lugares altos

121-He sentido miedo sin motivo

81-Me he sentido aturdido o confundido

14-Tiendo a sentirme nervioso

19-Me he sentido angustiado, con los nervios de punta

043-Me he sentido agitado o alterado

013-Generalmente me siento cómodo, sosegado y apacible

001-Con facilidad me siento asustado, con temor o miedo

021-He sentido que me falta el aire o me ahogo

051-He sentido dolor o presión en el pecho.

074-Me siento inseguro, como si me fuera a caer.

045-Tengo sensaciones de adormecimiento $\mathrm{u}$ hormigueo en el cuerpo

079-Tengo escalofríos frecuentemente.
$4,33(1,67-7,00)$

0,4277

0,8877

$4,74(2,11-7,36)$

0,5570

0,8837

$7,12(4,42-9,82)$

0,5508

0,8839

$4,02(1,39-6,65)$

0,5092

0,8850

$3,31(0,60-6,02)$

0,5755

0,8830

$4,41(2,07-6,75)$

0,4455

0,8875

$4,60(1,80-7,40)$

0,6514

0,8804

$4,76(2,04-7,47)$

0,6465

0,8806

4,46 (1,60-7,32)

0,6333

0,8811

$5,20(2,42-7,98)$

0,6774

0,8794

$5,18(2,53-7,84)$

0,5980

0,8823

$3,97(1,32-6,63)$

0,6186

0,8815

$4,81(2,10-7,52)$

0,6187

0,8815

$3,70(0,89-6,51)$

0,6099

0,8819

$3,84(1,20-6,49)$

0,5640

0,8835

$5,03(2,33-7,73)$

0,6304

0,8811

$5,46(3,01-7,90)$

0,5057

0,8855

$4,18(1,29-7,06)$

0,5102

0,8848 
Construcción y propiedades psicométricas de la Escala de Ansiedad de Lima de 20 ítems (EAL-20).

Tabla 3. Análisis factorial policórico de la escala final de 20 ítems. $\mathrm{AF}=$ ansiedad física; $\mathrm{AP}=$ ansiedad psíquica; $\mathrm{FS}=$ fobia social; $\mathrm{Ag}=$ agorafobia.

\begin{tabular}{|c|c|c|c|c|c|c|c|c|c|c|}
\hline$\underline{\text { Factores } \rightarrow}$ & 1 & 2 & & & 3 & & & 4 & & \\
\hline \multirow{2}{*}{$\begin{array}{l}\text { Eigenvalue } \\
\text { Ítem }\end{array}$} & \multirow{2}{*}{$\frac{9,66}{1}$} & \multicolumn{2}{|c|}{2,68} & \multicolumn{3}{|c|}{1,13} & \multicolumn{4}{|c|}{0,94} \\
\hline & & $\begin{array}{c}1 \\
\mathrm{AF}+\mathrm{AP}\end{array}$ & $\begin{array}{c}2 \\
\text { Fobia }\end{array}$ & $\begin{array}{c}1 \\
\mathrm{AF}+\mathrm{AP}\end{array}$ & $\begin{array}{c}2 \\
\text { FS }\end{array}$ & $\begin{array}{c}3 \\
\mathrm{Ag}\end{array}$ & $\begin{array}{c}1 \\
\mathrm{AF}\end{array}$ & $\begin{array}{c}2 \\
\text { FS }\end{array}$ & $\begin{array}{c}3 \\
\mathrm{AP}\end{array}$ & $\begin{array}{c}4 \\
\mathrm{Ag}\end{array}$ \\
\hline 84-Tengo miedo de conocer gente nueva & 0,56 & $-*$ & 0,91 & - & 0,88 & - & - & 0,89 & - & - \\
\hline $\begin{array}{l}\text { 118-Tengo miedo de dar exámenes o } \\
\text { tener una entrevista de trabajo }\end{array}$ & 0,69 & - & 0,84 & - & 0,82 & - & - & 0,78 & 0,43 & - \\
\hline $\begin{array}{l}\text { 034-Me es difícil hablar con otras } \\
\text { personas, tengo temor, me cuesta iniciar } \\
\text { una conversación o no se me ocurre qué } \\
\text { decir }\end{array}$ & 0,49 & - & 0,78 & - & 0,78 & - & - & 0,79 & - & - \\
\hline $\begin{array}{l}\text { 080-Me incomoda que me observen } \\
\text { cuando trabajo, escribo, camino o tengo } \\
\text { algún objeto que se me puede caer. }\end{array}$ & 0,66 & - & 0,77 & - & 0,79 & - & - & 0,76 & - & - \\
\hline $\begin{array}{l}\text { 088-Tengo miedo de hacer el ridículo y } \\
\text { sentirme humillado o avergonzado }\end{array}$ & 0,67 & - & 0,80 & - & 0,83 & - & - & 0,81 & - & - \\
\hline 119-Me da miedo alejarme de mi casa & 0,64 & 0,44 & 0,47 & - & - & 0,76 & - & - & - & 0,71 \\
\hline $\begin{array}{l}\text { 75-Me incomodaría entrar o salir de un } \\
\text { lugar lleno de gente. }\end{array}$ & 0,69 & - & 0,71 & - & 0,64 & 0,45 & - & 0,68 & - & 0,52 \\
\hline 127-Me da miedo estar en lugares altos & 0,52 & 0,54 & - & - & - & 0,73 & - & - & - & 0,69 \\
\hline 121-He sentido miedo sin motivo & 0,78 & 0,76 & - & 0,62 & - & 0,52 & - & - & 0,74 & - \\
\hline 81-Me he sentido aturdido o confundido & 0,78 & 0,73 & - & 0,71 & - & - & 0,52 & - & 0,58 & - \\
\hline 14-Tiendo a sentirme nervioso & 0,81 & 0,75 & - & 0,67 & - & - & 0,53 & - & 0,52 & - \\
\hline $\begin{array}{l}\text { 19-Me he sentido angustiado, con los } \\
\text { nervios de punta }\end{array}$ & 0,81 & 0,75 & - & 0,67 & - & - & 0,51 & - & 0,55 & - \\
\hline 043-Me he sentido agitado o alterado & 0,72 & 0,74 & - & 0,80 & - & - & 0,67 & - & 0,45 & - \\
\hline $\begin{array}{l}\text { 013-Generalmente me siento cómodo, } \\
\text { sosegado y apacible }\end{array}$ & 0,75 & 0,64 & - & 0,59 & - & - & - & - & 0,61 & - \\
\hline $\begin{array}{l}\text { 001-Con facilidad me siento asustado, } \\
\text { con temor o miedo }\end{array}$ & 0,75 & 0,65 & - & 0,51 & - & 0,52 & - & - & 0,78 & - \\
\hline $\begin{array}{l}\text { 021-He sentido que me falta el aire o } \\
\text { me ahogo }\end{array}$ & 0,72 & 0,84 & - & 0,81 & - & - & 0,75 & - & - & - \\
\hline $\begin{array}{l}\text { 051-He sentido dolor o presión en el } \\
\text { pecho. }\end{array}$ & 0,66 & 0,79 & - & 0,80 & - & - & 0,80 & - & - & - \\
\hline $\begin{array}{l}\text { 074-Me siento inseguro, como si me } \\
\text { fuera a caer. }\end{array}$ & 0,77 & 0,71 & - & 0,59 & - & 0,46 & 0,55 & - & - & 0,42 \\
\hline $\begin{array}{l}\text { 045-Tengo sensaciones de } \\
\text { adormecimiento u hormigueo en el } \\
\text { cuerpo }\end{array}$ & 0,60 & 0,71 & - & 0,74 & - & - & 0,79 & - & - & - \\
\hline 079-Tengo escalofríos frecuentemente. & 0,69 & 0,74 & - & 0,72 & - & - & 0,76 & - & - & - \\
\hline$\%$ varianza explicada & 48,78 & 36,89 & 25,43 & 30,91 & 23,6 & 13,53 & 22,93 & 22,29 & 17,91 & 9,67 \\
\hline
\end{tabular}

${ }^{*}$ Los valores $<0,4$ se han omitido. 
Tabla 4. Puntajes de la EAL-20 según nivel de severidad de la ansiedad calificado con la CGI-S.

\begin{tabular}{lccc}
\hline $\begin{array}{l}\text { CGI-S } \\
\text { Ansiedad }\end{array}$ & Media & DE & Frecuencia \\
\hline 1= normal & 5,33 & 6,42 & 9 \\
2= mínima & 6,37 & 5,09 & 35 \\
3= leve & 7,25 & 4,97 & 76 \\
4= moderada & 11,41 & 4,83 & 110 \\
5= marcada & 12,96 & 3,67 & 23 \\
6= grave & 16,00 & 0,00 & 1 \\
Total & 9,41 & 5,45 & 254 \\
\hline
\end{tabular}

Tabla 5. Sensibilidad, especificidad e índice de Youden para cada punto de corte de la EAL-20 y área bajo la curva ROC, tomando como patrón de referencia un CGI-S $\geq 4$.

\begin{tabular}{cccc}
\hline $\begin{array}{c}\text { Punto de } \\
\text { corte }\end{array}$ & Sensibilidad & Especificidad & $\begin{array}{c}\text { Índice de } \\
\text { Youden }\end{array}$ \\
\hline$(\geq 0)$ & 1 & 0 & 0 \\
$(\geq 1)$ & 0,9851 & 0,0667 & 0,0518 \\
$(\geq 2)$ & 0,9776 & 0,2083 & 0,1859 \\
$(\geq 3)$ & 0,9701 & 0,2667 & 0,2368 \\
$(\geq 4)$ & 0,9627 & 0,3417 & 0,3044 \\
$(\geq 5)$ & 0,9403 & 0,3833 & 0,3236 \\
$(\geq 6)$ & 0,8806 & 0,4667 & 0,3473 \\
$(\geq 7)$ & 0,8209 & 0,5333 & 0,3542 \\
$(\geq 8)$ & 0,7612 & 0,5667 & 0,3279 \\
$(\geq 9)$ & 0,7313 & 0,6083 & 0,3396 \\
$(\geq 10)$ & 0,694 & 0,675 & 0,369 \\
$(\geq 11)$ & 0,6343 & 0,75 & 0,3843 \\
$(\geq 12)$ & 0,5746 & 0,7917 & 0,3663 \\
$(\geq 13)$ & 0,4627 & 0,8417 & 0,3044 \\
$(\geq 14)$ & 0,403 & 0,8833 & 0,2863 \\
$(\geq 15)$ & 0,3358 & 0,8917 & 0,2275 \\
$(\geq 16)$ & 0,291 & 0,95 & 0,241 \\
$(\geq 17)$ & 0,1567 & 0,9583 & 0,115 \\
$(\geq 18)$ & 0,097 & 0,9667 & 0,0637 \\
$(\geq 19)$ & 0,0224 & 1 & 0,0224 \\
$(\geq 20)$ & 0,0075 & 1 & 0,0075 \\
$(>20)$ & 0 & 1 & 0 \\
\hline Área bajo la curva ROC: $0,7569(\mathrm{IC}-95 \%: 0,6979-0,8160)$
\end{tabular}

somáticos y agorafobia (8). En dicho estudio y en el proceso de construcción de la EAL-20 se evidencia la superposición entre la ansiedad física y el pánico.

La ansiedad psíquica de la EAL-20 incluye síntomas tales como sentirse nervioso, angustiado, asustado, agitado, alterado, aturdido, confundido, con temor y con miedo sin motivo; y no sentirse cómodo, sosegado o apacible. El concepto de ansiedad psíquica es cercano al de distrés el cual además de manifestaciones de ansiedad (nerviosismo e inquietud) incluye algunos síntomas centrales de la depresión (tristeza, desesperanza, minusvalía y falta de energía)(9). De acuerdo al estudio de Wright y cols. (10) el distrés involucra síntomas de depresión, ansiedad generalizada y ataques de pánico. El FourDimensional Symptom Questionnaire (4DSQ) incluye una subescala de distrés (11) que evalúa síntomas tales como tristeza, preocupación, alteraciones del sueño, falta de energía, tensión, irritabilidad, desinterés, incapacidad para afrontar las cosas y dificultad para pensar con claridad.

La dimensión de ansiedad física está constituida por los síntomas de disnea, molestias en el pecho, sensación de inestabilidad, parestesias y escalofríos. En nuestro estudio, dolor o presión en el pecho fue el que tuvo la carga factorial más alta dentro de los síntomas físicos. Una de las 4 dimensiones del 4DSQ es la de síntomas físicos, dentro de la cual los síntomas más vinculados a dicha dimensión son los cardiovasculares (11). Asimismo, dentro de los síntomas físicos de la Escala de Ansiedad de Hamilton, los más importantes son los cardiovasculares (12).

El factor de fobia social incluye el temor a hablar con otras personas, conocer gente nueva, ser observado haciendo alguna actividad, dar exámenes o tener una entrevista de trabajo, hacer el ridículo y sentirse humillado o avergonzado. El ítem de la EAL-20 sobre el temor a conocer gente nueva es el más importante de la dimensión de fobia social y es similar al ítem 12 de la Escala de Ansiedad Social de Liebowitz referido a "conocer gente extraña" que tiene la carga factorial más alta en la dimensión número 1 denominada "interacción social" (13). Además, el factor de fobia social de la EAL-20 concuerda con el contenido de los tres ítems de la Escala Breve de Ansiedad Social (mini-SPIN) constituido por: "el miedo a la vergüenza me hace evitar hacer cosas o hablar con las personas", "evito las actividades en donde soy el centro de atención" y "sentir vergüenza de parecer estúpido es uno de mis peores miedos"(14); y también con el del Social Phobia Screener (SOPHS) conformado por los siguientes 5 items: “¿Hasta qué punto te has sentido temeroso o avergonzado de cualquier situación social durante el mes pasado?”, “¿El miedo o la vergüenza que experimentó durante el mes pasado fue excesivo o irrazonable?", "Durante el mes pasado, 
¿ha evitado alguna situación social debido a su miedo o vergüenza?", "Durante el mes pasado, ¿cuánto ha sufrido a través de alguna situación social debido a su miedo o vergüenza?" y "Durante el mes pasado, ¿cuánto se ha interrumpido su trabajo, su hogar o su vida social debido a su miedo o vergüenza?"(15).

El factor agorafobia de la EAL-20 involucra 3 ítems: el miedo a alejarse de casa, entrar o salir de un lugar lleno de gente y estar en lugares altos. De acuerdo a un estudio psicométrico del Mobility Inventory (MI), la agorafobia tiene 3 dimensiones: evitación de espacios públicos, evitación de espacios cerrados y evitación de espacios abiertos. El ítem de la EAL-20 sobre miedo a lugares altos corresponde a la dimensión evitación de espacios abiertos del MI. El ítem de la EAL-20 sobre miedo a alejarse de casa tiene un ítem similar en el MI que se relaciona a las dimensiones de evitación de espacios abiertos y evitación de espacios públicos. Finalmente el ítem sobre lugares llenos de gente de la EAL-20 corresponde a la dimensión de evitación de espacios públicos (16). Por otra parte, el ítem referido a los lugares llenos de gente se asemeja al ítem único para la detección de agorafobia del Visual Screener for Common Mental Disorders, un instrumento administrado por computadora; dicho ítem cuyo texto se acompaña de imágenes de situaciones en las que hay gente aglomerada, es: " $¿$ Tiene miedo de lugares donde hay mucha gente y de donde es difícil salir rápidamente?" (17).

En el modelo unifactorial de la EAL-20, los ítems "tiendo a sentirme nervioso" y "me he sentido angustiado, con los nervios de punta", pertenecientes a la dimensión de "ansiedad psíquica", son los que mayores cargas factoriales han tenido; en congruencia con esto tenemos que el ítem sobre sentirse "nervioso" o "ansioso" es uno de los 2 ítems de la GAD-2, una escala muy breve constituida por los ítems nucleares de la GAD-7, y ambas escalas han demostrado ser útiles en pacientes de atención primaria para la detección del trastorno de ansiedad generalizada y otros trastornos relacionados tales como el de pánico, la fobia social y el de estrés postraumático (18).

En el modelo de 2 factores, se identifica una dimensión de fobias (fobia social + agorafobia) y otra de ansiedad psíquica + física. La fusión entre fobia social y agorafobia es compatible con la fuerte relación y comorbilidad que hay entre estas dos condiciones $(8,19)$. La sintomatología de fobia social y agorafobia está estrechamente relacionada e incluida en el concepto de temor $(10,20)$.
Considerando 3 factores, la fobia social puede separarse de la agorafobia, permaneciendo juntas la ansiedad psíquica y la somática, que recién se diferencian al explorarse la estructura de 4 factores.

Aunque el punto de corte con mayor J para identificar individuos con ansiedad de intensidad moderada o más es $\geq 11(\mathrm{~J}=0,38)$, este valor tiene una diferencia importante entre sensibilidad $(63,4 \%)$ y especificidad (75\%) por lo que sugerimos usar $\geq 10$ como punto de corte considerando que es el que tiene mayor equilibrio entre sensibilidad y especificidad y no hay mucha diferencia con el J máximo ( $\mathrm{s}=69,4 \%$, $\mathrm{e}=67,5 \%, \mathrm{~J}=0,37)$. Comparativamente la EAL-130 con un punto de corte de $\geq 60$ tiene valores similares: $\mathrm{s}=69,4 \%, \mathrm{e}=68,3 \%, \mathrm{~J}=0,38)(3)$.

Dentro de las limitaciones del estudio, debemos considerar que los individuos incluidos son pacientes de consulta externa de psiquiatría con trastornos mentales sin psicosis o alteraciones cognitivas, sería importante tener datos del desempeño de la escala en sujetos de la población general en los que la mayoría no presentan patología mental y evaluar su capacidad para identificar en ellos la presencia de trastornos de ansiedad.

En conclusión, se redujo la escala de ansiedad inicial de 130 ítems a una de 20 ítems (EAL-20); la cual posee una buena consistencia interna y consta de 4 dimensiones: ansiedad psíquica, síntomas físicos, fobia social y agorafobia. Se sugiere usar un punto de corte de $\geq 10$ como un indicador de ansiedad moderada o de mayor severidad.

Conflictos de interés: Los autores declaran no tenerlos. Declaración de financiamiento: Financiado por los autores.

\section{Correspondencia:}

Antonio Lozano Vargas

Jirón Puente y Cortez 599 Magdalena del Mar. Lima, Perú.

Celular: (+51)999374904

Correo electrónico: antoniolv2000@yahoo.com / edward.lozano.v@upch.pe

\section{REFERENCIAS BIBLIOGRÁFICAS}

1. Metzler DH, Mahoney D, Freedy JR. Anxiety Disorders in Primary Care. Prim Care. 2016;43: 245261. 
2. Instituto Nacional de Salud Mental. Estudio Epidemiológico de Salud Mental en Lima Metropolitana y Callao Replicación 2012. Informe General. Anales de Salud Mental. 2013;29: 1-392.

3. Lozano-Vargas A, Vega-Dienstmaier J. Evaluación psicométrica y desarrollo de una versión reducida de la nueva escala de ansiedad en una muestra hospitalaria de Lima, Perú. Rev Peru Med Exp Salud Publica. 2013;30: 212-219.

4. Vega-Dienstmaier JM. Construcción de una nueva escala en español para medir ansiedad. Rev Neuropsiquiatr. 2011;74: 230-241.

5. American Psychiatric Association. Diagnostic and Statistical Manual of Mental Disorders. 5th ed. Arlington, VA: American Psychiatric Publishing; 2013.

6. Guy W. Clinical Global Impressions Scale. In: Rush JA, Task Force for the Handbook of Psychiatric Measures, editors. Handbook of Psychiatric Measures. Washington, DC: American Psychiatric Association; 2000.

7. Angulo M, Rooks BT, Gill M, Goldstein T, Sakolsky D, Goldstein B, et al. Psychometrics of the Screen for Adult Anxiety Related Disorders (SCAARED)- A new scale for the assessment of DSM-5 anxiety disorders. Psychiatry Res. 2017;253: 84-90.

8. Vega-Dienstmaier JM. Estructura de los síndromes afectivos y ansiosos en pacientes psiquiátricos ambulatorios de un hospital general. Lima: Tesis de Maestría en Epidemiología Clínica, Universidad Peruana Cayetano Heredia; 2017.

9. Prochaska JJ, Sung H-Y, Max W, Shi Y, Ong M. Validity study of the K6 scale as a measure of moderate mental distress based on mental health treatment need and utilization. Int J Methods Psychiatr Res. 2012;21: 88-97.

10. Wright AGC, Krueger RF, Hobbs MJ, Markon KE, Eaton NR, Slade T. The structure of psychopathology: toward an expanded quantitative empirical model. J Abnorm Psychol. 2013;122: 281-294.

11. Terluin B, Smits N, Brouwers EPM, de Vet HCW. The Four-Dimensional Symptom Questionnaire (4DSQ) in the general population: scale structure, reliability, measurement invariance and normative data: a cross-sectional survey. Health Qual Life Outcomes. 2016;14: 130.

12. Porter E, Chambless DL, McCarthy KS, DeRubeis
RJ, Sharpless BA, Barrett MS, et al. Psychometric properties of the reconstructed Hamilton depression and anxiety scales. J Nerv Ment Dis. 2017;205(8):656664. doi:10.1097/NMD.0000000000000666

13. Safren SA, Heimberg RG, Horner KJ, Juster HR, Schneier FR, Liebowitz MR. Factor structure of social fears: The Liebowitz Social Anxiety Scale. J Anxiety Disord. 1999;13: 253-270.

14. Wiltink J, Kliem S, Michal M, Subic-Wrana C, Reiner I, Beutel ME, et al. Mini - social phobia inventory (mini-SPIN): psychometric properties and population based norms of the German version. BMC Psychiatry. 2017;17: 377.

15. Batterham PJ, Mackinnon AJ, Christensen H. Community-Based Validation of the Social Phobia Screener (SOPHS). Assessment. 2017;24: 958-969.

16. Rodriguez BF, Pagano ME, Keller MB. Psychometric characteristics of the Mobility Inventory in a longitudinal study of anxiety disorders: replicating and exploring a three component solution. J Anxiety Disord. 2007;21: 752-761.

17. van Ballegooijen W, Riper H, Donker T, Martin Abello K, Marks I, Cuijpers P. Single-item screening for agoraphobic symptoms: validation of a web-based audiovisual screening instrument. PLoS One. 2012;7: e38480.

18. Kroenke K, Spitzer RL, Williams JBW, Monahan PO, Löwe B. Anxiety disorders in primary care: prevalence, impairment, comorbidity, and detection. Ann Intern Med. 2007;146: 317-325.

19. Kessler RC, Chiu WT, Demler O, Merikangas KR, Walters EE. Prevalence, severity, and comorbidity of 12-month DSM-IV disorders in the National Comorbidity Survey Replication. Arch Gen Psychiatry. 2005;62: 617-627.

20. Krueger RF. The structure of common mental disorders. Arch Gen Psychiatry. 1999;56: 921-926.

Recibido: $25 / 11 / 2018$

Aceptado: 14/12/2018 